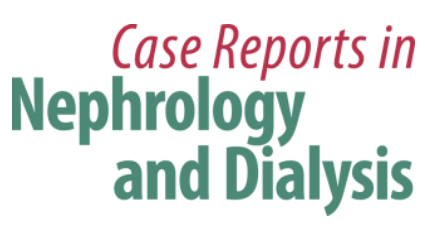

\title{
Improvement in Gemcitabine-Induced Thrombotic Microangiopathy with Rituximab in a Patient with Ovarian Cancer: Mechanistic Considerations
}

\author{
Sangeetha Murugapandian ${ }^{a} \quad$ Babitha Bijin $^{a}$ Iyad Mansour ${ }^{b}$ \\ Sepehr Daheshpour ${ }^{\mathrm{b}}$ Biju G. Pillai ${ }^{\mathrm{a}}$ Bijin Thajudeen ${ }^{\mathrm{a}}$ \\ Abdulla K. Salahudeen ${ }^{a}$ \\ Departments of ${ }^{a}$ Nephrology and ${ }^{b}$ Medicine, University of Arizona Medical Center, \\ Tucson, Ariz., USA
}

\section{Key Words}

Gemcitabine $\cdot$ Thrombotic microangiopathy $\cdot$ Rituximab $\cdot$ Ovarian cancer

\begin{abstract}
Gemcitabine is a potent and widely used anticancer drug. We report a case of gemcitabineinduced thrombotic microangiopathy (GCI-TMA), a known but not widely recognized complication of gemcitabine use, and our experience of treating GCI-TMA with rituximab. A 74year-old woman was referred to our clinic for an evaluation of worsening renal function. She has recently been treated for ovarian cancer (diagnosed in 2011) with surgery (tumor debulking and bilateral salpingo-oophorectomy) along with cisplatin chemotherapy in 2012, followed by carboplatin/doxorubicin in 2013 and recent therapy for resistant disease with gemcitabine. Laboratory tests showed anemia, normal platelets and elevated lactate dehydrogenase. A peripheral smear revealed numerous schistocytes, and a kidney biopsy showed acute as well as chronic TMA. The patient continued on gemcitabine therapy, and treatment with plasma exchange was started. Since there was no response to treatment even after 5 sessions of plasma exchange, one dose of rituximab was given, which was associated with a drop in the creatinine level to $2 \mathrm{mg} / \mathrm{dl}$. The pathogenesis of renal injury could be the effect of direct injury to the endothelium mediated by cytokines. Usual treatment includes withdrawing the drug and initiation of treatment with plasmapheresis with or without steroids. In cases resistant to plasmapheresis, treatment with rituximab can be tried. The mechanism of action of rituximab might be due to the reduced production of B-cell-dependent cytokines that
\end{abstract}

KARGER 125/s $\quad \begin{aligned} & \text { Bijin Thajudeen, MD } \\ & \text { University of Arizona Medical Center } \\ & 1501 \text { N Campbell Ave } \\ & \text { Tucson, AZ } 85724 \text { (USA) } \\ & \text { E-Mail bijint@gmail.com }\end{aligned}$




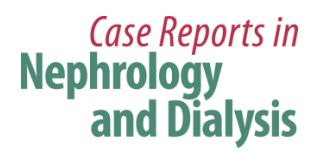

Case Rep Nephrol Dial 2015;5:160-167

DOI: $10.1159 / 000435807$

C) 2015 S. Karger AG, Basel

www.karger.com/cnd

Murugapandian et al.: Improvement in GCI-TMA with Rituximab in a Patient with Ovarian Cancer: Mechanistic Considerations

drive endothelial dysfunction by depleting $B$ cells. Patients receiving gemcitabine chemotherapy should be monitored for the development of TMA, and early treatment with plasma exchange along with rituximab might benefit these patients who already have a bad prognosis.

(c) 2015 S. Karger AG, Basel

\section{Introduction}

Thrombotic microangiopathy (TMA) encompasses a spectrum of diseases characterized by microangiopathic hemolytic anemia, thrombocytopenia and acute renal failure [1]. Several medications, including gemcitabine, have been implicated in the pathogenesis of TMA. Various treatment options have been tried for this condition, with varying degrees of treatment response. Here, we describe a case of gemcitabine-induced TMA with atypical presentation treated with rituximab.

\section{Case Report}

A 74-year-old Caucasian female was referred to our nephrology clinic for an evaluation of worsening renal function. Her past medical history was significant for multiple sclerosis, gastroesophageal reflux disease and ovarian cancer. She had undergone tumor debulking as well as bilateral salpingo-oophorectomy in 2011. Following surgery, she had been treated with platinum-based chemotherapy with cisplatin, which was completed in 2012. In 2013, she had a relapse of the disease (clinically as well as an elevation in tumor marker CA-125), for which platinum-based chemotherapy was repeated using carboplatin and doxorubicin. She was resistant to this treatment following which gemcitabine monotherapy was initiated in 2013. After the fifth cycle of gemcitabine therapy (cumulative dose of 9,460 mg), her creatinine level started increasing (to $7.3 \mathrm{mg} / \mathrm{dl}$ from a baseline of $1.1 \mathrm{mg} / \mathrm{dl}$ ). Her other medications included ondansetron, pantoprazole and multivitamins. Her family history was positive only for hypertension. She denied any history of smoking, drug abuse or alcohol use.

On physical examination, her vital signs were stable, with a temperature of $98^{\circ} \mathrm{F}$, a blood pressure of 162/100 $\mathrm{mm} \mathrm{Hg}$, a pulse rate of 78 beats/min and a respiratory rate of 18 breaths/min. There was no rash or edema. Her conjunctiva was pale but anicteric. Cardiac examination demonstrated a systolic murmur, normal heart sounds and no pericardial rub. The rest of the examination was unremarkable.

Laboratory test results at the time of initial evaluation are shown in table 1 . The urine sediment showed granular casts. There were no dysmorphic red blood cells or cellular casts. Her urine dipstick revealed 4+ proteinuria, and her urine protein-to-creatinine ratio was 2.4 $\mathrm{g} / \mathrm{g}$ creatinine. A peripheral smear showed schistocytes, which was consistent with microangiopathic hemolytic anemia. Renal ultrasound was unremarkable. In view of the drop in hemoglobin level, elevated lactate dehydrogenase (LDH), schistocytes in the peripheral smear, new-onset hypertension and acute kidney injury, the possibility of TMA was considered. A complement study showed a C3 level of $113 \mathrm{mg} / \mathrm{dl}$ (range 82-185) and a C4 level of $19 \mathrm{mg} / \mathrm{dl}$ (range 15-53). Antinuclear antibodies, anti-dsDNA, hepatitis panel, HIV, anti-Scl70 , antineutrophil cytoplasmic antibody and anticardiolipin AB were negative. A renal biopsy was performed, which showed glomeruli with mesangial lysis and endothelial cell swelling. There was entrapment of small red blood cell fragments within the endothelial cell cytoplasm. Silver staining showed irregularities in the glomerular basement membrane including frequent duplication. No definite intracapillary thrombus was noted. There was patchy 


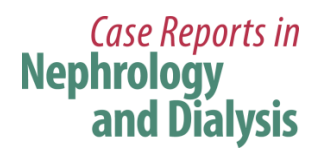

Case Rep Nephrol Dial 2015;5:160-167

DOI: $10.1159 / 000435807$

07

(C) 2015 S. Karger AG, Basel

www.karger.com/cnd

Murugapandian et al.: Improvement in GCI-TMA with Rituximab in a Patient with Ovarian Cancer: Mechanistic Considerations

mononuclear interstitial inflammation. An immunofluorescence study was unremarkable. Electron microscopy showed capillary loops with swollen endothelium, occlusion of the lumen with cells, cell debris and electron-dense material (possibly fibrin). There was focal duplication of the basement membrane and diffuse effacement of epithelial cell foot cytoplasm (fig. 1, 2). These findings were consistent with acute as well as with chronic TMA. Since gemcitabine has been considered as a cause of TMA and there were no other etiologies for TMA in this patient, the final diagnosis was TMA secondary to gemcitabine. A normal ADAMTS13 and an antibody test to ADAMTS13 ruled out thrombotic thrombocytopenic purpura.

Treatment with plasma exchange was started, but the creatinine level remained unchanged even after 5 daily sessions. Hence, the decision was made to start rituximab. One single dose of rituximab $(1 \mathrm{~g})$ was given. There was gradual improvement in renal function over the next few weeks. The creatinine level at 2 months after treatment with rituximab was $2 \mathrm{mg} / \mathrm{dl}$. There was no change in LDH (in fact, LDH increased over time likely due to the cancer). A repeat peripheral smear after 1.5 months of treatment with rituximab did not demonstrate schistocytes. The patient did not receive any further doses of gemcitabine. Since the ovarian cancer continued to progress relentlessly, the patient decided to go on hospice care. The trend in serum creatinine levels over time is represented in figure 3.

\section{Discussion}

Gemcitabine is a pyrimidine antimetabolite that was introduced in 1987 for the treatment of solid tumors [2]. The first case of gemcitabine-related TMA was reported in 1994 [3]. The incidence varies between 0.008 and $0.078 \%$ [3]. The standard treatment of TMA varies from simple drug discontinuation and supportive care to the use of plasmapheresis, steroids or hemodialysis [2]. Despite the use of aggressive treatment, this disease process generally has a bad prognosis [2].

The pathogenesis of TMA is believed to be endothelial damage, which leads to platelet aggregation, fibrin formation and thrombus deposition [4]. There are various postulates for how the endothelium is injured in cases of TMA. The role of immune complexes has been described in the pathogenesis of TMA: formed as a result of the underlying disease process, they trigger aggregation and deposition of platelets around areas of toxin-related endothelial damage in the kidney microvasculature [5]. The role of cytokines in the pathogenesis of endothelial injury has also been discussed. Various cytokines like interferons might be involved [6]. These cytokines might inhibit vascular endothelial cell growth factor (VEGF) in glomerular endothelial cells [7]. VEGF plays a major role in maintaining vascular integrity by protecting the endothelial cells [7]. TMA has been reported with the use of anti-VEGF drugs like sunitinib [8]. Other postulated mechanisms include generation of the ADAMTS13 inhibitor and abnormal immune response to the von Willebrand factor-cleaving protease $[9,10]$. In our case, the absence of immunofluorescence in renal biopsy makes an immune complex theory less likely. Similarly, normal ADAMTS13 and antibody to ADAMTS13 do not support the involvement of ADAMTS13. Hence, direct endothelial damage from the drug leading to platelet aggregation, fibrin formation and thrombus formation is the most likely possibility. There are multiple reports of drug-induced TMA (especially mitomycin C) and the role of direct endothelial injury described in the literature [10]. Some of the histopatholgical findings seen in our case might support this view. In an animal study on rats treated with mitomycin, the earliest injury noted was endothelial damage. Subsequently, at 1 week of exposure to mitomycin, many glomerular capillary lumens were reduced or obliterated by plate- 


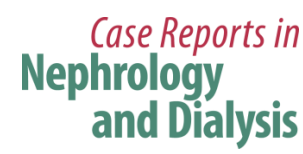

Case Rep Nephrol Dial 2015;5:160-167

DOI: 10.1159/000435807

(c) 2015 S. Karger AG, Basel

www.karger.com/cnd

Murugapandian et al.: Improvement in GCI-TMA with Rituximab in a Patient with Ovarian Cancer: Mechanistic Considerations

lets, mononuclear cells and swollen endothelium. In addition, at 1 month, there was extensive obliteration of foot process of the visceral epithelium, mesangial interposition in the basement membrane (splitting of the basement membrane) and mononuclear inflammation of the interstitium [11]. In our case, there were similar histopathological findings (electron microscopy), which might favor the role of direct endothelial injury with gemcitabine. This might also prove the role of progressive damage to glomeruli following the initial insult with gemcitabine. The onset of clinically evident disease is often delayed, frequently occurring months after chemotherapy has been discontinued [2]. The initial insult might have happened way earlier, and changes in biochemical parameters might have occurred once the injury is well established. The cumulative effect of glomerular endothelial damage might also play a role in the development of clinically evident TMA [2].

There are previous reports of gemcitabine-induced TMA reported in the literature [2]. Our case is unique due to absence of marked hemolysis and thrombocytopenia, as previous cases presented with all the classical features including thrombocytopenia and hemolysis. In this case, although hemolysis was evidenced by the presence of schistocytes and a mild elevation in $\mathrm{LDH}$, other features like a dramatic drop in hemoglobin level, a low haptoglobin level and elevated unconjugated hyperbilirubinemia were absent. Moreover, thrombocytopenia was also absent. In this context, it is notable that drug-induced TMA may occur in the absence of other systemic features including microangiopathic hemolytic anemia or thrombocytopenia [12]. Hence, in the absence of clinical suspicion, these cases might be missed, resulting in inadvertent outcomes. Affected patients typically present with slowly progressive renal failure, new or exacerbated hypertension and relatively bland urine sediment. Proteinuria and microscopic hematuria are also frequently reported [2].

This case is also unique because of the response to treatment with rituximab. Rituximab is a monoclonal antibody that selectively targets CD20-positive B cells and has been used in the treatment of TMA resulting from gemcitabine [2]. In our case, rituximab was started after the lack of a clinical response to plasmapheresis. The exact mechanism by which rituximab improves TMA is not fully elucidated and there are different theories about how the use of rituximab may result in the improvement in TMA. Rituximab might act as an immune modulator in controlling the disease, especially in scenarios where immune complexes or antibodies to ADAMTS13 might be playing a role in the pathogenesis. There might be a mechanism similar to that seen in patients treated with rituximab for rheumatoid arthritis. The depletion of B cells may reduce the production of B-cell-dependent cytokines that drive endothelial dysfunction [13-15]. In our case, this might be the most likely mechanism for improvement in renal function with rituximab treatment although the contribution of plasma exchange in the form of a late response could not be completely ruled out. The standard definition for refractory disease in disorders like thrombotic thrombocytopenic purpura (non-response to 7 daily plasma exchange treatments) may not be applicable to all TMAs (moreover, our patient had only received 5 days of plasma exchange therapy) [16].

\section{Conclusion}

Although a randomized, controlled trial of rituximab in GCI-TMA may be difficult due to the rarity of this complication, more case reports like ours with a salutary effect may underscore the possibility of using rituximab as a first-line or alternative treatment strategy for patients with TMA secondary to gemcitabine. These are patients who already have a poor prognosis, and the development of renal failure may further increase the risk of mortality. Hence, it is essential to early identify renal toxicity and initiate appropriate intervention, 
Murugapandian et al.: Improvement in GCI-TMA with Rituximab in a Patient with Ovarian Cancer: Mechanistic Considerations

including the discontinuation of gemcitabine therapy, thereby improving survival even if only for a few weeks or months.

\title{
Disclosure Statement
}

\author{
All authors report no conflicts of interest.
}

\section{References}

1 Halevy D, Radhakrishnan J, Markowitz G, Appel G: Thrombotic microangiopathies. Crit Care Clin 2002;18:309-320, vi.

2 Bharthuar A, Egloff L, Becker J, George M, Lohr JW, Deeb G, Iyer RV: Rituximab-based therapy for gemcitabine-induced hemolytic uremic syndrome in a patient with metastatic pancreatic adenocarcinoma: a case report. Cancer Chemother Pharmacol 2009;64:177-181.

-3 Izzedine H, Isnard-Bagnis C, Launay-Vacher V, Mercadal L, Tostivint I, Rixe O, Brocheriou I, Bourry E, Karie S, Saeb S, Casimir N, Billemont B, Deray G: Gemcitabine-induced thrombotic microangiopathy: a systematic review. Nephrol Dial Transplant 2006;21:3038-3045.

4 von Baeyer H: Plasmapheresis in thrombotic microangiopathy-associated syndromes: review of outcome data derived from clinical trials and open studies. Ther Apher 2002;6:320-328.

5 Gutterman LA, Kloster B, Tsai HM: Rituximab therapy for refractory thrombotic thrombocytopenic purpura. Blood Cells Mol Dis 2002;28:385-391.

6 Mahe J, Meurette A, Moreau A, Vercel C, Jolliet P: Renal thrombotic microangiopathy caused by interferon beta-1a treatment for multiple sclerosis. Drug Des Devel Ther 2013;7:723-728.

7 Kuenen BC, Levi M, Meijers JC, van Hinsbergh VW, Berkhof J, Kakkar AK, Hoekman K, Pinedo HM: Potential role of platelets in endothelial damage observed during treatment with cisplatin, gemcitabine, and the angiogenesis inhibitor SU5416. J Clin Oncol 2003;21:2192-2198.

8 Bollée G, Patey N, Cazajous G, Robert C, Goujon JM, Fakhouri F, Bruneval P, Noël LH, Knebelmann B: Thrombotic microangiopathy secondary to VEGF pathway inhibition by sunitinib. Nephrol Dial Transplant 2009;24:682-685.

-9 Furlan M, Robles R, Galbusera M, et al: von Willebrand factor-cleaving protease in thrombotic thrombocytopenic purpura and the hemolytic-uremic syndrome. N Engl J Med 1998;339:1578-1584.

10 Shah G, Yamin H, Smith H: Mitomycin-C-induced TTP/HUS treated successfully with rituximab: case report and review of the literature. Case Rep Hematol 2013;2013:130978.

11 Cattell V: Mitomycin-induced hemolytic uremic kidney. An experimental model in the rat. Am J Pathol 1985;121:88-95.

12 Pham PT, Peng A, Wilkinson AH, et al: Cyclosporine and tacrolimus-associated thrombotic microangiopathy. Am J Kidney Dis 2000;36:844-850.

13 Benucci M, Saviola G, Manfredi M, Sarzi-Puttini P, Atzeni F: Factors correlated with improvement of endothelial dysfunction during rituximab therapy in patients with rheumatoid arthritis. Biologics 2013;7:69-75.

14 Gonzalez-Juanatey C, Llorca J, Vazquez-Rodriguez TR, Diaz-Varela N, Garcia-Quiroga H, Gonzalez-Gay MA: Short-term improvement of endothelial function in rituximab treated rheumatoid arthritis patients refractory to tumor necrosis factor alpha blocker therapy. Arthritis Rheum 2008;59:1821-1824.

-15 Hsue PY, Scherzer R, Grunfeld C, Imboden J, Wu Y, Del Puerto G, Nitta E, Shigenaga J, Schnell Heringer A, Ganz P, Graf J: Depletion of B-cells with rituximab improves endothelial function and reduces inflammation among individuals with rheumatoid arthritis. J Am Heart Assoc 2014;3:e001267.

16 Scully M, Hunt BJ, Benjamin S, Liesner R, Rose P, Peyvandi F, Cheung B, Machin SJ: Guidelines on the diagnosis and management of thrombotic thrombocytopenic purpura and other thrombotic microangiopathies. Br J Haematol 2012;158:323-335. 
Murugapandian et al.: Improvement in GCI-TMA with Rituximab in a Patient with Ovarian Cancer: Mechanistic Considerations

Table 1. Laboratory tests at the time of initial evaluation

\begin{tabular}{lrc}
\hline Laboratory results & Value & Reference range \\
\hline Hemoglobin, g/dl & 9.9 & $11.5-15.5$ \\
White blood count, $\times 10^{9} / \mathrm{l}$ & 5.2 & $3.4-10.4$ \\
Platelets, $\times 10^{9} / \mathrm{l}$ & 233 & $150-425$ \\
Haptoglobin, mg/dl & 95 & $14-35$ \\
Fibrinogen, mg/dl & 176 & $200-430$ \\
Prothrombin time, INR & 2.5 & \\
Partial thromboplastin time, s & 44.3 & $29-35$ \\
Sodium, mmol/l & 139 & $136-145$ \\
Potassium, mmol/l & 5.3 & $3.5-5.1$ \\
Chloride, mmol/l & 112 & $101-111$ \\
Bicarbonate, mmol/l & 20 & $20-29$ \\
Urea nitrogen, mg/dl & 65 & $7-20$ \\
Phosphorus, mg/dl & 3.7 & $2.3-4.7$ \\
Creatinine kinase, IU/l & 113 & $29-168$ \\
Total bilirubin, mg/dl & 0.6 & $0.2-1.2$ \\
Alanine transaminase, IU/l & 15 & $0-55$ \\
Aspartate transaminase, IU/l & 23 & $5-34$ \\
Alkaline phosphatase, IU/l & 79 & $125-243$ \\
Albumin, g/dl & 2.5 & $3.4-4.8$ \\
LDH, IU/l & 324 & $125-243$ \\
Lactic acid, mmol/l & 1.3 & $0.5-2.2$ \\
CA-125, IU/ml & 513 & $0-35$ \\
Uric acid, mg/dl & 7.8 & $4-6$ \\
\hline
\end{tabular}

INR = International normalized ratio. 


\section{Case Reports in \\ Nephrology and Dialysis}

\begin{tabular}{l|l}
\hline \multicolumn{2}{l}{ Case Rep Nephrol Dial 2015;5:160-167 } \\
\hline DOI: 10.1159/000435807 & $\begin{array}{l}\text { ○ 2015 S. Karger AG, Basel } \\
\text { www.karger.com/cnd }\end{array}$ \\
\hline
\end{tabular}

Murugapandian et al.: Improvement in GCI-TMA with Rituximab in a Patient with Ovarian Cancer: Mechanistic Considerations

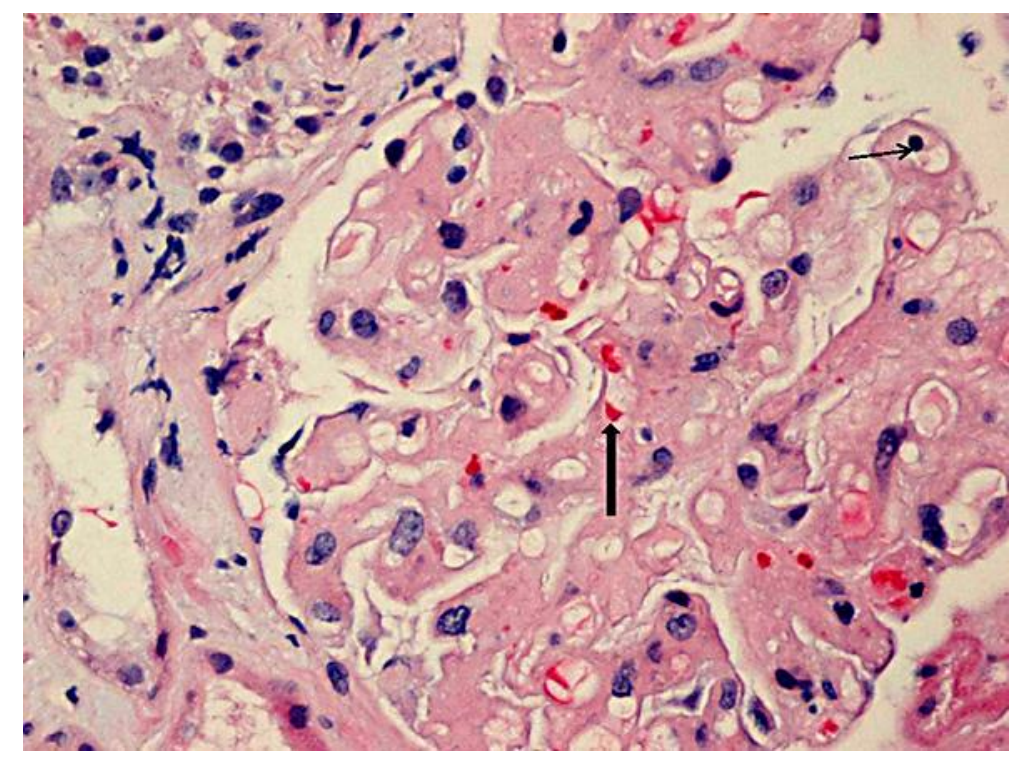

Fig. 1. Light microscopy (H\&E stain) showing mesangiolysis, entrapment of small red blood cell fragments within the glomerular endothelial cell cytoplasm (thick arrow), reactive endothelial cells (thin arrow) and splitting of the basement membrane.

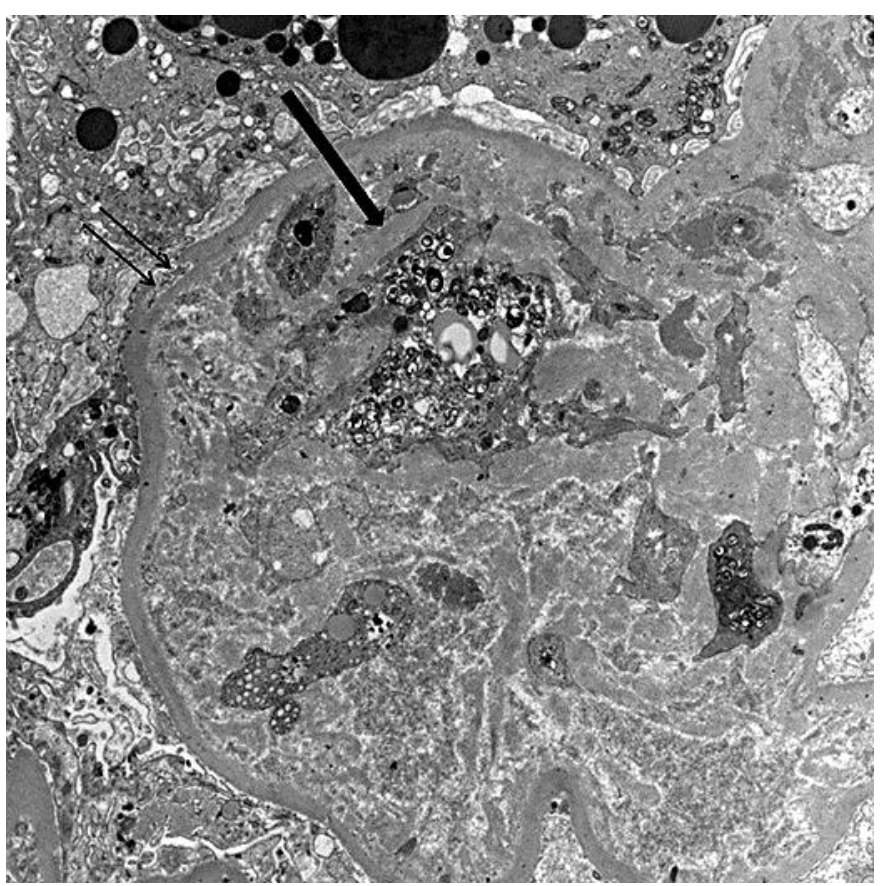

Fig. 2. Electron microscopy showing capillary loops with swollen endothelium (thick arrow), occlusion of the lumen with cells, cell debris and electron-dense material (possibly fibrin). There is focal duplication of the basement membrane (double thin arrow) and diffuse effacement of epithelial cell foot cytoplasm. 
Case Reports in

Nephrology

and Dialysis
Case Rep Nephrol Dial 2015;5:160-167

DOI: 10.1159/000435807

\begin{tabular}{l|l} 
& $\begin{array}{l}\text { C } 2015 \text { S. Karger AG, B } \\
\text { www.karger.com/cnd }\end{array}$ \\
\hline
\end{tabular}

Murugapandian et al.: Improvement in GCI-TMA with Rituximab in a Patient with Ovarian Cancer: Mechanistic Considerations

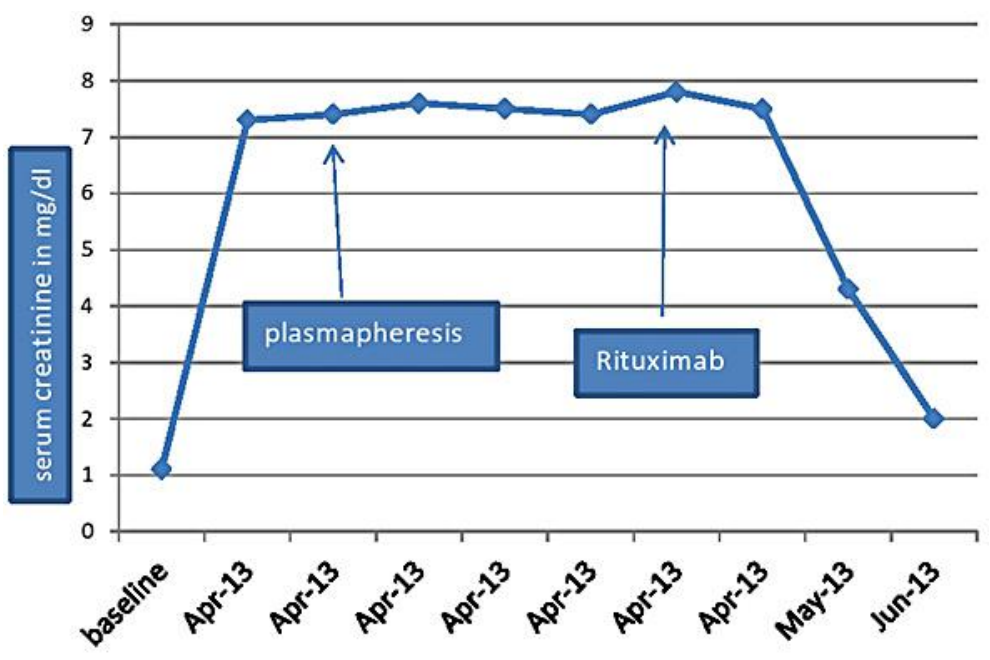

Fig. 3. Trend in creatinine levels over time. Arrows point towards the time of initiation of plasmapheresis and rituximab. 